\title{
ASSESSMENT OF MYOCARDIAL VIABILITY USING LOW DOSE DOBUTAMINE THREE DIMENSIONAL SPECKLE TRACKING STRESS TRANSTHORACIC ECHOCARDIOGRAPHY
}

\author{
By \\ Bassam Mohammed Mehkiemer*, Ahmed Al-Habbaa, Mustafa \\ Mukarrab and Ali Al-Amin \\ Department of Cardiology, Faculty of Medicine, Al-Azhar University, Cairo, Egypt \\ *Corresponding author: Bassam M Mekhiemer, \\ E-mail address: Bassamcvs@gmail.com
}

\begin{abstract}
Background: Three-dimensional speckle tracking echocardiography (3D STE) is used recently to assess many cardiac diseases; it is considered as natural growth of two-dimensional speckle tracking echocardiography (2D STE). The LV mechanics is in nature a 3D phenomenon and its accurate assessment requires a 3D imaging method. 3DSTE has been implemented for measuring 3D strain, and has emerged as a more physiologically sound tool for analyzing the complexity of LV mechanics, overcoming the inherent limitations of 2D STE.
\end{abstract}

Objective: To determine the relative accuracy of area strain measured by 3D speckle-tracking echocardiography combined with low dose dobutamine for the detection of myocardial viability before coronary revascularization in comparison with MPI.

Patients and Methods: The study included 40 patients referred to Bab El-She'riya University Hospital nuclear lab for assessment of myocardial viability by MPI before coronary revascularization. Then all patients were referred to Islamic Cardiac Center Echo lab where complete conventional transthoracic echocardiography was done followed by Low dose dobutamine (LDD) stress transthoracic echocardiography with 3D STE then comparing results of 3D strain of viable segments with that of nonviable segments and with corresponding segments in MPI studies.

Results: The study showed that 241 segments in the 40 patients were assigned as abnormal segments, 114 were viable and 127 were nonviable, resting area strain (AS)was $-18.7 \pm 2.5$ for viable segments comparing to $-12.6 \pm 5.3$ for non-viable segment. A cut-off point of -16 was chosen to differentiate viable segments from non-viable ones with sensitivity $80.75 \%$ and specificity $83.1 \%$. The average LDD AS value for viable segments was- $24.5 \pm 2.7$ and was $-15.2 \pm 4.8$ of non-viable segments with a cut-off point of -24 to differentiate viable segments from non-viable ones with sensitivity of $84.75 \%$ and a specificity of $89.1 \%$. The average increase of AS of viable segments after LDD was $-5.8 \pm 2.3$ with a cut-off point to detect viability was a -0.24 increase in LDD from resting AS with a sensitivity of $95.75 \%$ and a specificity of $98.1 \%$.

Conclusion: AS obtained from 3D STE may be used clinically as an indicator to detect myocardial viability with higher sensitivity and specificity when combined with LDD.

Keywords: Area Strain (AS), three dimensional (3D), speckle tracking echocardiography (STE), myocardial viability.

Abbreviations: AS: area strain- CS: circumferential strain - LS: longitudinal strain- RS: radial strain- 3D: three dimension - 3DSTE: there dimension speckle tracking echocardiography - MPI: myocardial perfusion image - LDD: low dose dobutamine - LDDSE: low dose dobutamine stress echocardiography. 


\section{INTRODUCTION}

After myocardial infarction, both morbidity and mortality depend upon the extent of left ventricular (LV) systolic dysfunction. Not all dysfunctional myocardial segments are due to necrosis which is non-viable and cause irreversible damage; also hibernating and stunned segment which are viable and reversibly losing their contractile function (Ryan and Perera, 2018). As for the hibernating or stunned myocardium, its contractile function may be recovered after revascularization; thus there is muchimproved LV function and a higher proportion of survival in patients with ischemic cardiomyopathy (whose myocardium may be viable) than in those with large areas of nonviable or scarred myocardium (Pegg et al., 2010).

Myocardial viability may be assessed by various methods that test the integrity of a number of cellular mechanisms of the viable cardiac myocytes. These mechanisms include the determination of maintained cell membrane integrity, preserved metabolic machinery, recruitable inotropic reserve (or contractile reserve), coronary micro vascular integrity and the absence of late enhancement. Viable myocardium is capable of augmenting its function in the presence of inotropic stimulus - a property called contractile reserve or recruitable inotropic reserve. The improvement in function of viable myocardium can be visualized either with echocardiography or other imaging techniques, like magnetic resonance imaging (Adrián and Christopher, 2018).

Dobutamine stress echocardiography is an established method for the assessment of coronary artery disease with a sensitivity and specificity of $80 \%-85 \%$ comparable to those of radio-isotopic myocardial perfusion studies (Allman, 2013).

2D STE when combined with LDD stress echocardiography in assessing myocardial viability it increases the sensitivity, specificity and accuracy of echocardiography in comparison to late gadolinium enhanced cardiac MRI (Wan, 2016).

Three-dimensional (3D) speckletracking echocardiography (3DSTE) is an advanced imaging technique designed for left ventricular (LV) myocardial deformation analysis based on 3D data sets. 3DSTE has the potential to overcome some of the intrinsic limitations of twodimensional STE (2DSTE) in the assessment of complex LV myocardial mechanics, offering additional deformation parameters (such as area strain) and a comprehensive quantitation of LV geometry and function from a single 3D acquisition. Albeit being a relatively young technique still undergoing technological developments, several experimental studies and clinical investigations have already demonstrated the reliability and feasibility of 3DSTE, as well as several advantages of 3DSTE over 2DSTE. This technique has provided new insights into LV mechanics in several clinical fields, such as the objective assessment of global and regional LV function in ischemic and non-ischemic heart diseases (Muraru et al., 2018).

The present work aimed to determine the relative accuracy of area strain measured by 3D speckle-tracking echocardiography combined with low 
dose dobutamine for the detection of myocardial viability before coronary revascularization in comparison with MPI.

\section{PATIENTS AND METHODS}

This study included 40 patients went to our Cardiology Department of Bab Alshe'ria University Hospital, in the period from April 2018 to- December 2019 for assessment of myocardial viability for possibility of myocardial revascularization. We exclude those with significant valvular heart disease as severe AS and severe MR and those with contraindication to dobutamine including unstable patients, such as those with decompensated heart failure or unstable angina, hypertrophic cardiomyopathy, arrhythmias that interfere with interpretation of dobutamine stress echocardiography or perfusion scintigraphy. Patients with bad echo window or when echo study protocol cannot be completed or who refuse to be included in the study all are excluded.

\section{All Subjects underwent the following:}

\section{Myocardial perfusion imaging (MPI):}

The entire 40 patients underwent myocardial single photon emission computed tomography using Tc-99m Sestamibi (Tc-99m-sestaMIBI SPECT). The studies were done in two settings one at rest and the second at rest with nitrate potentiation in the next day, Beta-blockers, calcium antagonists and nitrates were discontinued in patients at least 24 hours before the study. Computed tomography images were acquired one hour after infusion of the radiotracer. Image acquisition was achieved with a dual head gamma camera (Siemens) without attenuation or scatter correction, using a low energy, all-purpose collimator. Trans axial tomograms were reconstructed for each patient, at short-axis, horizontal and vertical long axis slices and were analyzed. A total of 17 myocardial segments per patient were studied.

\section{Conventional transthoracic echocardiographic assessment:}

Standard Echo-Doppler examinations were performed using a 2.5 multi frequency $1.7-4 \mathrm{MHz}$ transducer (GE Vivid 9 Ultrasound Machine). All the patients were examined in the left lateral decubitus position and echocardiographic images were acquired from the standard views (parasternal long-axis, parasternal short axis at papillary muscle level, apical four -chamber, apical five -chamber and apical two- chamber). Recordings and calculations of different cardiac chambers and ejection fractions were made according to the recommendations of the American Society of Echocardiography (Lang et al., 2015).

\section{Low Dose Dobutamine Stress Echocardiography (LDDSE):}

Beta-blockers, calcium antagonists and nitrates were discontinued in patients at least 48 hours before low dose dobutamine echocardiography. Low dose dobutamine (LDD) infusion was administered using automated infusion pump. Dobutamine was delivered intravenously using 3 minutes staged protocol starting from 5 $\mu \mathrm{g} / \mathrm{kg} / \mathrm{min}$ for three minutes, then 10 $\mu \mathrm{g} / \mathrm{kg} / \mathrm{min}$ for another three minutes period, then 3 minutes recovery 
without dobutamine. 3D echocardiographic acquisition of full volume scan was acquired from apical window (will be verified later on). The recorded 3D image loops were digitally stored at rest and at $10 \mu \mathrm{g} / \mathrm{kg} / \mathrm{min}$ LDD echocardiography for later offline analysis. Patients were continuously monitored by ECG and blood pressure measurement during the test.

\section{3 Dimension Speckle tracking echocardiography study (3D STE):}

A full-volume scan was acquired by harmonic imaging from an apical approach, using a frame rate (in volume per second) higher than $40 \%$ of the individual heart rate in order to increase the possibility that the "speckles" could be recognizable in successive frames. Accordingly, four electrocardiogram-gated consecutive beats were acquired while holding the breath in expiration. The quality of acquisition was verified in each patient, before storing the volume data set, by selecting a 12-slice display mode available on the machine to ensure the entire LV cavity and wall were included in the full volume. Data sets were stored digitally in raw data format and equipped with commercially available software (4D Auto LVQ software), for analysis of LV volumes, EF, LV mass, and 4D STE deformation parameters. LV analysis was performed according to a previously described methodology (Muraru et al., 2010).

\section{RESULTS}

Table (1): Demographic and risk factors data among studied group:

\begin{tabular}{|c|c|c|c|}
\hline \multirow{2}{*}{ Factors Variables } & \multicolumn{2}{c|}{$\begin{array}{c}\text { Frequency } \\
(\mathrm{N}=40)\end{array}$} & Percent \\
\hline Age & \multicolumn{2}{|c|}{ Mean \pm SD: 56.1 \pm 9.7 } \\
\hline Male & \multicolumn{2}{|c|}{9} & $\mathbf{7 7 . 5 \%}$ \\
\hline Female & Negative & 19 & 47.5 \\
\hline \multirow{2}{*}{ HTN } & Positive & 21 & 52.5 \\
\cline { 2 - 4 } & Negative & 22 & 55 \\
\cline { 2 - 4 } & Positive & 18 & 45 \\
\hline \multirow{3}{*}{ Dyslipidemia } & Negative & 16 & 40 \\
\cline { 2 - 4 } & Positive & 24 & $\mathbf{6 0}$ \\
\hline \multirow{2}{*}{ Smoking } & Negative & 13 & 32.5 \\
\cline { 2 - 4 } & Positive & 27 & 67.5 \\
\hline \multirow{2}{*}{ Family history } & Irrelevant & 21 & 52.5 \\
\cline { 2 - 4 } & Relevant & 19 & 47.5 \\
\hline
\end{tabular}


Table (2): Conventional TTE measurements:

\begin{tabular}{|c|c|c|c|c|c|c|}
\hline \multicolumn{7}{|c|}{ Descriptive Statistics } \\
\hline & \multicolumn{7}{|c|}{ Range } & Mean & \pm & SD \\
\hline LVIDD & $\mathbf{4 . 8 0 0}$ & - & $\mathbf{6 . 9 0 0}$ & $\mathbf{5 . 8 3 0}$ & \pm & $\mathbf{0 . 4 8}$ \\
\hline LVISD & $\mathbf{3 . 0 0 0}$ & - & $\mathbf{4 . 7 0 0}$ & $\mathbf{3 . 9 6 2}$ & \pm & $\mathbf{0 . 4 4}$ \\
\hline LVEDV & $\mathbf{1 0 0 . 0 0 0}$ & - & $\mathbf{1 9 0 . 0 0 0}$ & $\mathbf{1 4 5 . 1 6 0}$ & \pm & $\mathbf{1 3 . 4 4 0}$ \\
\hline LVESV & $\mathbf{4 0 . 0 0 0}$ & - & $\mathbf{9 5 . 0 0 0}$ & $\mathbf{4 3 . 6 4 0}$ & \pm & $\mathbf{1 0 . 8 3 0}$ \\
\hline EF & $\mathbf{2 9 . 0 0 0}$ & - & $\mathbf{5 8 . 0 0 0}$ & $\mathbf{3 9 . 6 0 0}$ & \pm & $\mathbf{7 . 4 2 0}$ \\
\hline
\end{tabular}

Percentage of viable and non-viable segments by MPI: A Total of 241 segments in the 40 patients were assigned as abnormal segments (viable or nonviable) by MPI with nitrate potentiation, 114 out of 241 segments revealed viability while 127 segments were assigned as non-viable, The number of viable and non-viable segments as identified by MPI after nitrate potentiation using MPI as a gold standard to distinguish between viable and non-viable segments ( Table 3)

\begin{tabular}{|c|c|c|}
\hline & Number & Percent \\
\hline Viable & $\mathbf{1 1 4}$ & $\mathbf{4 7 . 3 \%}$ \\
\hline Non-viable & $\mathbf{1 2 7}$ & $\mathbf{5 2 . 7 \%}$ \\
\hline Total & $\mathbf{2 4 1}$ & $\mathbf{1 0 0 \%}$ \\
\hline
\end{tabular}

3D speckle tracking echocardiography reading Mean \pm SD of different segments grouped by MPI readings both during rest and after LDD and mean difference between resting and LDD of viable and nonviable segments showing that comparing to MPI for viability detection, AS measured by 3 D STE is highly sensitive and specific tool in detecting myocardial viability especially when adding LDD. ( Table 4)

\begin{tabular}{|c|c|c|c|c|}
\hline & \multicolumn{2}{|c|}{$3 \mathrm{D}$ speckle tracking Echo } & \multicolumn{2}{|c|}{$\begin{array}{c}\text { Difference between resting and } \\
\text { LDD }\end{array}$} \\
\hline & & Mean value \pm SD & Mean difference \pm SD & P value \\
\hline \multirow[t]{2}{*}{ AS Viable } & Resting & $-18.7 \pm 2.5$ & \multirow[t]{2}{*}{$5.806 \pm 2.3-$} & \multirow[t]{2}{*}{$<0.0001$} \\
\hline & LDD & $-24.5 \pm 2.7$ & & \\
\hline \multirow[t]{2}{*}{ AS Non-viable } & Resting & $-12.6 \pm 5.3$ & \multirow[t]{2}{*}{$2.838 \pm \mathbf{3 . 1 -}$} & \multirow[t]{2}{*}{$<0.0001$} \\
\hline & LDD & $-15.2 \pm 4.8$ & & \\
\hline \multirow[t]{2}{*}{ LS Viable } & Resting & $-13.3 \pm 5.3$ & \multirow[t]{2}{*}{$4.054 \pm 2.9-$} & \multirow[t]{2}{*}{$<0.002$} \\
\hline & LDD & $-17.5 \pm 1$ & & \\
\hline \multirow[t]{2}{*}{ LS Non-viable } & Resting & $-9.8 \pm 3.7$ & \multirow[t]{2}{*}{$2.013 \pm 2.1-$} & \multirow[t]{2}{*}{$<0.001$} \\
\hline & LDD & $-11.1 \pm 3.8$ & & \\
\hline \multirow[t]{2}{*}{ CS Viable } & Resting & $-15.8 \pm 1.5$ & \multirow[t]{2}{*}{$-2.000 \pm 1.7$} & \multirow[t]{2}{*}{0.034} \\
\hline & LDD & $-18.1 \pm 1.2$ & & \\
\hline \multirow[t]{2}{*}{ CS Non-viable } & Resting & $-10.6 \pm 3.1$ & \multirow[t]{2}{*}{$.892 \pm 2.04-$} & \multirow[t]{2}{*}{0.04} \\
\hline & LDD & $-12.02 \pm 3.5$ & & \\
\hline \multirow[t]{2}{*}{ RS Viable } & Resting & $15.82 \pm 1.3$ & \multirow[t]{2}{*}{$1.287 \pm \mathbf{1 . 3}$} & \multirow[t]{2}{*}{0.12} \\
\hline & LDD & $17.12 \pm 1.1$ & & \\
\hline \multirow[t]{2}{*}{ RS Non-viable } & Resting & $9.54 \pm 2.3$ & \multirow[t]{2}{*}{$0.595 \pm 0.9$} & \multirow[t]{2}{*}{0.6} \\
\hline & LDD & $10.16 \pm 2.5$ & & \\
\hline
\end{tabular}




\section{DISCUSSION}

In our study we found that AS obtained from 3D STE may be used clinically as an indicator to detect myocardial viability with higher sensitivity and specificity when combined with LDD. The classic methodological gold standard for detecting myocardial viability has been positron emission tomography (PET). Since this technique is costly and not widely available, another most widely applied nuclear technique which is singlephoton emission computed tomography (SPECT) utilizing the tracers 201Thallium, 99mTechnetium sestamibi, or $99 \mathrm{mTechnetium} \mathrm{tetrofosmin} \mathrm{has} \mathrm{long}$ been used to evaluate viability. Besides these nuclear studies, the most widespread technique to assess regional myocardial viability and the potential for functional recovery has been dobutamine echocardiography (Mansour and Zhong hua 2014).

Dobutamine stress echocardiography is an established method for the assessment of coronary artery disease with a sensitivity and specificity of $80 \%-85 \%$ comparable to those of radio-isotopic myocardial perfusion studies (Allman 2013).

2D STE was approved in assessing regional wall motion abnormalities with longitudinal strain being the most sensitive parameter by detecting regional myocardial deformation. 2D STE when combined with LDD stress echocardiography in assessing myocardial viability it increases the sensitivity, specificity and accuracy of echocardiography in comparison to late gadolinium enhanced cardiac MRI (Wang, 2016).

3DSTE is being a relatively young technique still undergoing technological developments, several experimental studies and clinical investigations have already demonstrated the reliability and feasibility of 3DSTE, as well as several advantages of 3DSTE over 2DSTE. This technique has provided new insights into LV mechanics in several clinical fields, such as the objective assessment of global and regional LV function in ischemic and non-ischemic heart diseases (Muraru et al., 2018).

Several studies assessed normal individual 3D strain to detect reference values of 3D strain as Muraru et al., 2014 and Kleijn et al., 2013.

Wang and his Colleagues (2015) evaluate myocardial infarction size with three-dimensional speckle tracking echocardiography in comparison with single photon emission computed tomography and they find correlation between global 3D strain detected by 3DSTE and infarction size detected by MPI

3DSTE might have potential for detection of myocardial viability in patients with cardiac dysfunction due to MI. (Hong et al., 2016) who compare viable myocardial segments detected by MPI as a gold standard with strain of the segments detected by 2DSTE and 3DSTE to compare between the results of viable and non-viable segments.

Our study results wers generally in agreement with the mentioned studies that used 3DSTE facility. 


\section{CONCLUSION}

3D speckle tracking when added to LDD SE M increased the sensitivity and specificity of echocardiography in detection of myocardial viability and this when generally applied makes detection of viability more easy and decreases the cost and hazards of radiation exposure so.

\section{REFERENCES}

1. Ali A., Mohammed H., Yehia E., Ashraf A. and Mostafa T (2018): Assessment of normal values of Area Strain by using Three-dimensional speckle-tracking Echocardiography. The Egyptian Journal of Hospital Medicine. Vol. 73 (11), Page 7902-7907.

2. Allman, K.C (2013): Noninvasive assessment myocardial viability: Current status and future directions. J. Nucl. Cardiol. 20, 618-637.

3. Adrián I. Löffler and Christopher M. Kramer (2018): Myocardial viability testing to guide coronary revascularization. Interv Cardiol Clin. 2018 Jul; 7(3): 355-365.

4. Hong R, Ping-Yang $Z$, You-Xiang $Z$, Jian-Xin $Z$, Wen-Fang Wu, Jing $D$ and Xiao-Wu M (2016): Assessment of Left Ventricular Myocardial Viability by 3Dimensional Speckle-Tracking Echocardiography in Patients with Myocardial infarction J Ultrasound Med 2016; 35:1631-1638 | 0278-4297.

5. Kleijn SA, Pandian NG, Thomas JD, Perez de Isla L, Kamp O, Zuber $M$, Nihoyannopoulos P, Forster T, Nesser HJ, Geibel A, Gorissen $W$ and Zamorano JL (2015): Normal reference values of left ventricular strain using three-dimensional speckle tracking echocardiography: results from a multicentre study. Eur Heart J
Cardiovasc Imaging. 2015 Apr; 16(4):410-6.

6. Lang RM, Bierig M, Devereux RB, Flachskampf FA, Foster E, Pellikka PA, Picard MH, Roman MJ, Seward J, Shanewise JS, Solomon SD, Spencer KT, Sutton MS and Stewart WJ (2015): Recommendations for chamber quantification: a report from the American Society of Echocardiography's Guidelines and European Association of Echocardiography, a branch of the European Society of Cardiology. J Am Soc Echocardiogr. 2015 Dec; 18(12):1440-63.

7. Langeland S, Rabben SI, Heimdal A and Gérard $O$ (2010): 4D Strain: Validation of New 3D Speckle Tracking and Left Ventricular Tool in Simulated Echocardiographic Data. Moderated Poster Presentation at Euroecho 2010. Abstract; P658.

8. Mansour A. and Zhong-Hua Sun (2014): Diagnostic value of 18 F-FDG PET in the assessment of myocardial viability in coronary artery disease: A comparative study with $99 \mathrm{mTc}$ SPECT and chocardiography. J Geriatr Cardiol. 2014 Sep; 11(3): 229-236.

9. Muraru D, Badano LP, Piccoli G, Gianfagna P, Del Mestre L, Ermacora $D$ and Proclemer A (2010): Validation of a novel automated border-detection algorithm for rapid and accurate quantitation of left ventricular volumes based on three-dimensional echocardiography. Eur J Echocardiogr. 2010 May; 11(4):359-68.

10. Muraru D, Cucchini U, Mihăilă S, Miglioranza MH, Aruta P, Cavalli G, Cecchetto A, Padayattil-Josè S, Peluso D, Iliceto $S$ and Badano LP (2014): Left ventricular myocardial strain by three-dimensional speckle-tracking echocardiography in healthy subjects 


\section{BASSAM MOHAMMED MEHKIEMER et al.,}

reference values and analysis of their physiologic and technical determinants. J Am Soc Echocardiogr. 2014 Aug; 27(8):858-871.e1.

11. Muraru, D., Niero, A., RodriguezZanella, H., Cherata, D. and Badano, L. (2018): Three-dimensional speckletracking echocardiography: benefits and limitations of integrating myocardial mechanics with three-dimensional imaging. Cardiovascular diagnosis and therapy, 2018, 8(1), 101-117.

12. Pegg, T. J., Selvanayagam, J. B., Jennifer, J., Francis, J. M., Karamitsos, T. D., Dall'Armellina, E., Smith, K. L., Taggart, D. P. and Neubauer, S. (2010): Prediction of global left ventricular functional recovery in patients with heart failure undergoing surgical revascularisation, based on late gadolinium enhancement cardiovascular magnetic resonance. Journal of cardiovascular magnetic resonance; official journal of the Society for Cardiovascular Magnetic Resonance, 12(1), 56.
13. Ryan, M. J. and Perera, D. (2018): Identifying and Managing Hibernating Myocardium: What's New and What Remains Unknown? Current heart failure reports, 15(4), 214-223.

14. Wang, C., Han, S., Xu, T., Wang, F., Wang, X., Chen, J., Hu, C., and Li, D (2016): Evaluation of myocardial viability in old myocardial infarcted patients with CHF: delayed enhancement MRI vs. low-dose dobutamine stress speckle tracking echocardiography. American journal of translational research, 8(9), 3731-3743, 2016.

15. Wang $Q$, Zhang $C$, Huang $D$, Zhang $L$, Yang F, An X, Ouyang Q, Zhang M, Wang S, Guo J and Ji D (2015): Evaluation of myocardial infarction size with three-dimensional speckle tracking echocardiography: a comparison with single photon emission computed tomography. Int $\mathrm{J}$ Cardiovasc Imaging. 2015 Dec; 31(8):1571-81. 


\section{تقييم حيوية عضلة القلب بواسطة الموجات فوق الصوتية على الإئ

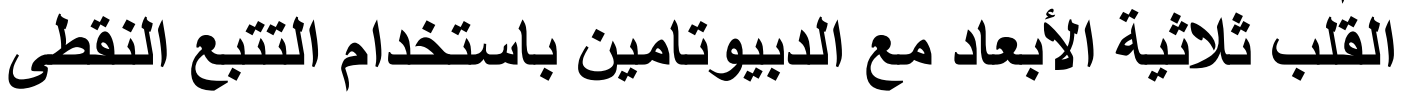

بسام محمد بسيوني زيدان مخيمر، على محمد الأمين عبد المجيد، مصطفى إبراهيم مقرب و أحمد محمد صلاح الدين أحمد علدان

قسم القلب والأوعية الدموية، كلية الطب، جامعة الأزهر، القاهرة (بنين)

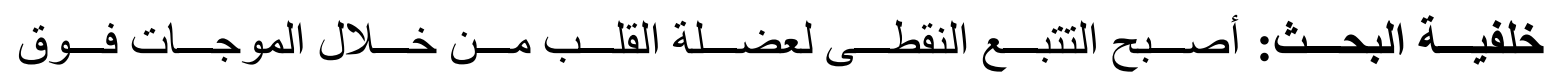

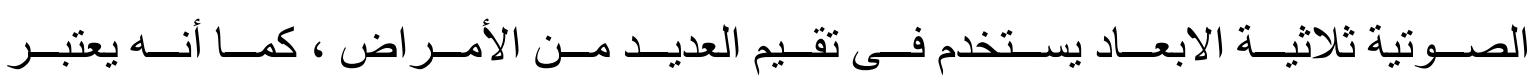

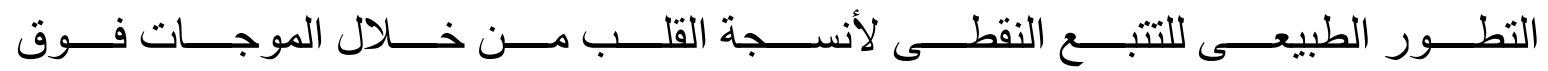

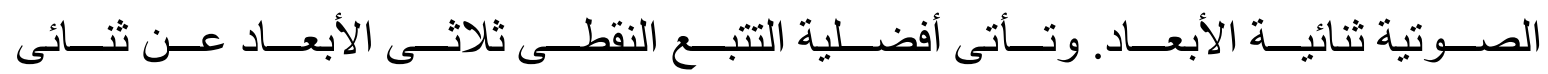

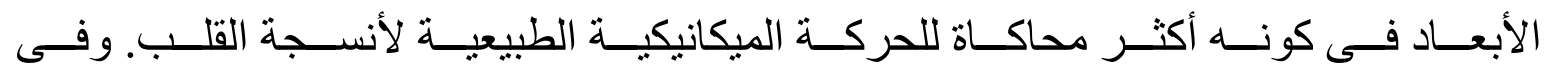

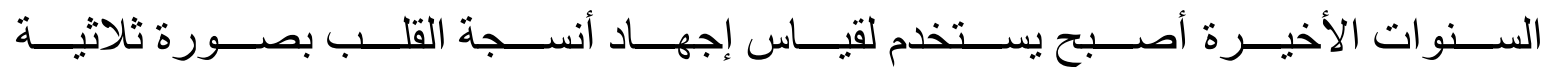

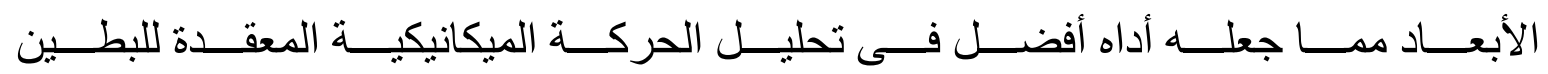

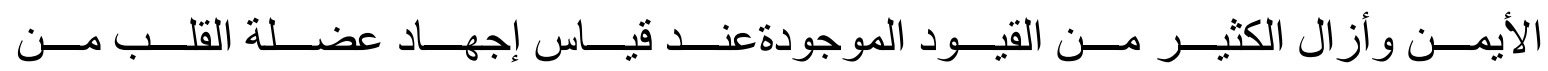
خلال التتبع النقطى ثنائى الأبعاد.

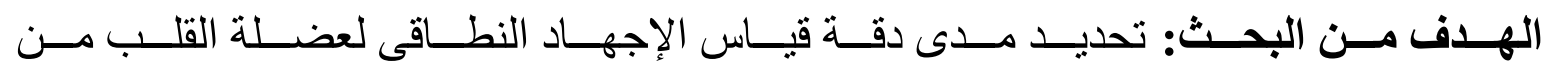

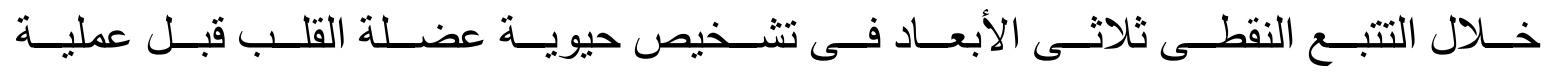

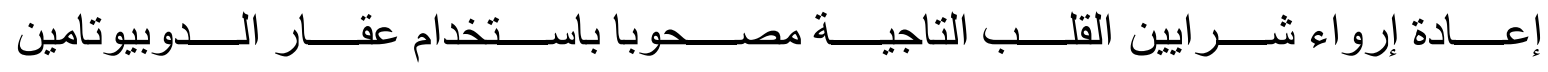
كمجهد دو ائى للقلب مقارنة بالمسح الذرى لعضلة القلب.

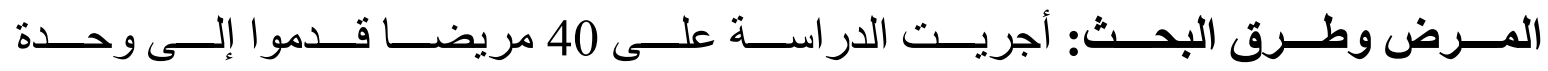

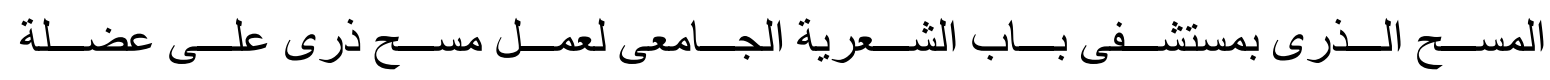

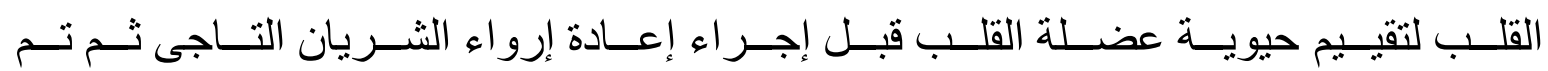

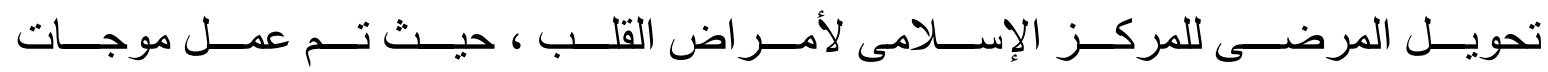

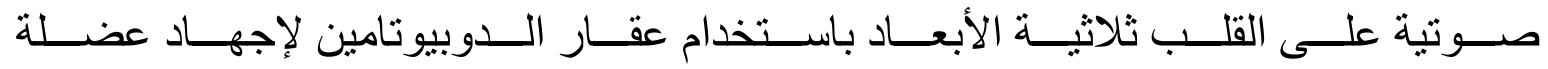

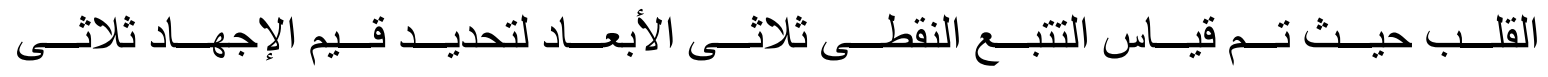




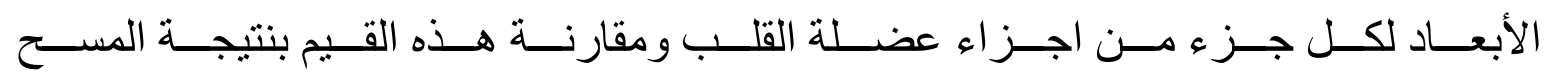
الذرى لكل قطعة مقابلة لها.

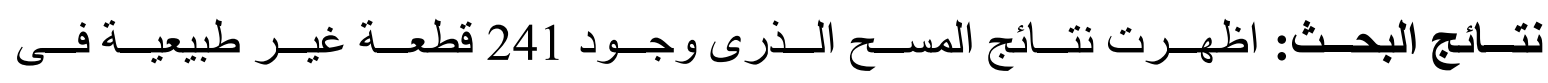

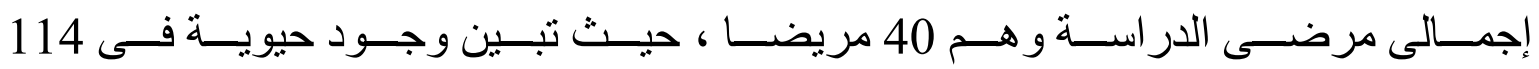

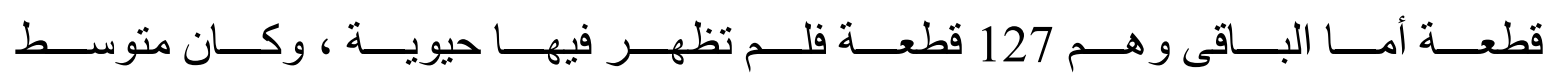

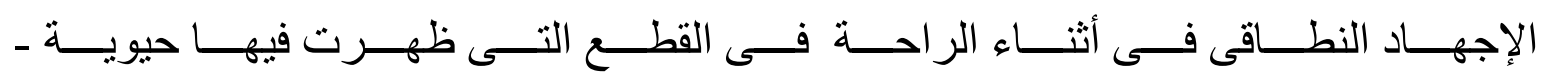

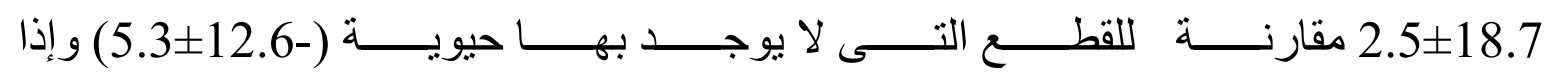

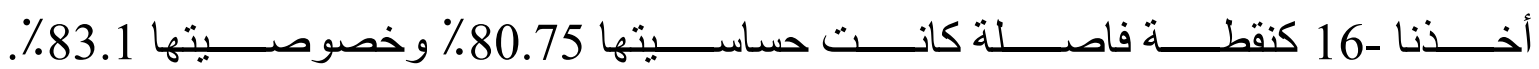

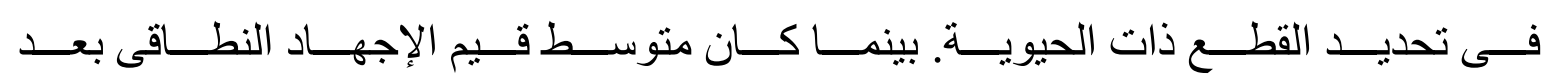

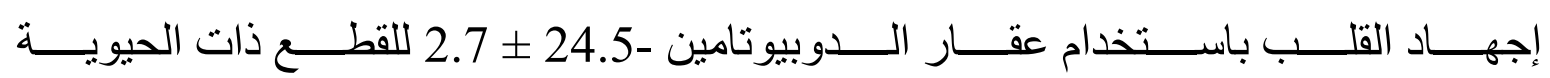

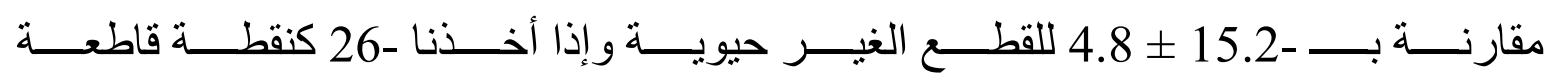

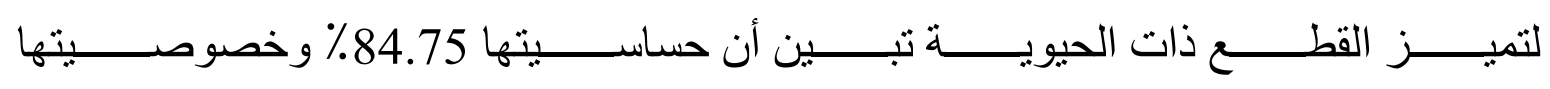

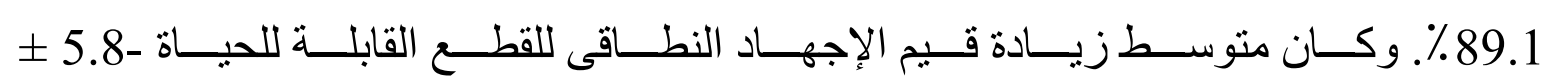

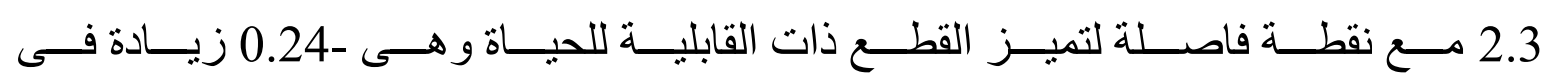

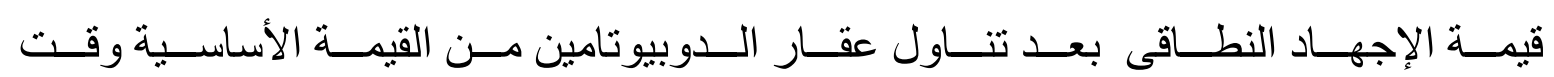
الراحة.

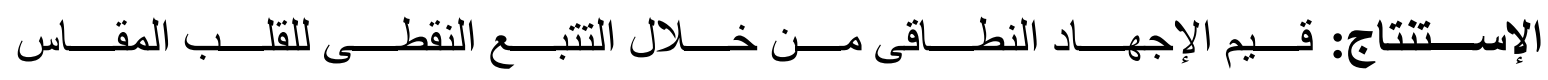

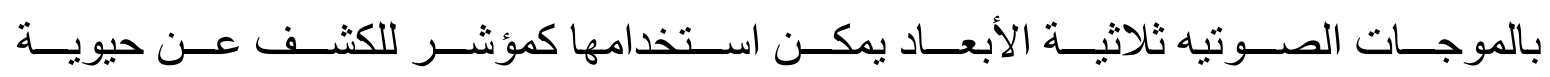

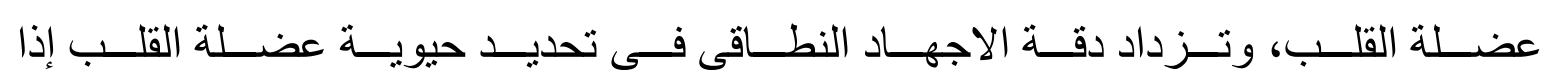
ما كان فحص القلب مصحوبا باستخدام الدوبيوتامين كعقار مجهد للقلب. 